\title{
Effects of oxygen concentration in IVM/IVF on marmoset monkey oocyte maturation and embryo development
}

\author{
Olena Y. Tkachenko ${ }^{1,2,7,8}$, Stefka Delimitreva ${ }^{1,3}$, Edris Wedi $^{1,4}$, Johanna U. Scheerer-Bernhard ${ }^{1,5}$, \\ Rodrigo R. Valle ${ }^{1,6}$, Penelope L. Nayudu ${ }^{1}$
${ }^{1}$ Reproductive Biomedicine Group, Reproductive Biology Unit, German Primate Centre, Leibniz Institute for Primate Research; Kellnerweg 4, Goettingen, 37077 Germany.
${ }^{2}$ Division of Reproductive and Developmental Sciences, Oregon National Primate Research Center, Oregon Health \& Science University, Beaverton, OR 97006, USA.
${ }^{3}$ Department of Biology, Medical University of Sofia; 1 St. Georgi Sofiyski Street, Sofia, 1431, Bulgaria. ${ }^{4}$ Gastroenterologie und GI Onkologie, Universitätsmedizin Göttingen, Robert-Koch-Straße 40, 37073 Göttingen.
${ }^{5}$ Department of Internal Medicine, Roskilde Hospital, Roskilde, 2100, Denmark.
${ }^{6}$ Instituto de Ciências da Saúde, Universidade Paulista, Health Sciences Institute, Paulista University, São Paulo, SP, Brazil.

\begin{abstract}
The effect of oxygen $\left(\mathrm{O}_{2}\right)$ concentration on in vitro development of the oocytes from antral follicles of naturally cycling common marmosets (Callithrix jacchus) has been investigated. Different $\mathrm{O}_{2}$ concentrations during in vitro maturation (20 or $8 \%)$ and during in vitro fertilization (20 or $5 \%$ ) have been applied in three combinations: $8-5,20-20,20-5$ (maturation and fertilization, respectively). Additionally, 8-20 conditions were tested in a pre-study, but since no fertilization occurred, this test group was excluded from further experiments. Oocyte maturation rate was significantly higher after IVM in $20 \%$ than in $8 \% \mathrm{O}_{2}$ (MI: $91 \%$ in $20-20 v s$. $78 \%$ in 8-5; MII: $69 v s$. $45 \%$ in $20-5$ and $50 \%$ in $8-5, \mathrm{P}<0.05$ for all). The lowest rate of embryo development occurred after maturation under $8 \% \mathrm{O}_{2}(59 \%$ in $8-5$ vs. $95 \%$ in $20-20$ and $83 \%$ in $20-5, \mathrm{P}<0.05$ for all), strongly suggesting this low tension may produce hypoxic conditions during maturation, which damages the oocytes. In conclusion, $20 \% \mathrm{O}_{2}$ was shown to be significantly superior to $8 \%$ $\mathrm{O}_{2}$ for marmoset oocyte maturation, and $5 \% \mathrm{O}_{2}$ was to be preferred over $20 \%$ for IVF, based on a trend for better embryo morphology and rates of progression.
\end{abstract}

Keywords: embryo, gas environment, in vitro fertilization, in vitro maturation, marmoset monkey, oocyte, oxygen.

\section{Introduction}

Optimal gas environment is an important physical factor influencing the success of oocyte and embryo culture. While reduced oxygen $\left(\mathrm{O}_{2}\right)$ concentration has been shown to be beneficial for embryo culture for a range of species (Bavister, 1995; Gelatt et al., 2001; Karja et al., 2004; Kovacic and Vlaisavljevic, 2008; Meintjes et al., 2009), $\mathrm{O}_{2}$ environment for in vitro maturation (IVM) and fertilization (IVF) reported as optimal for different species under various conditions is more variable. Protocols for IVM/IVF of various animal species, including human, which are currently applied by different laboratories, are based on both high and low oxygen tension for oocyte maturation and/or fertilization (Piotrowska-Nitsche et al., 2009; Koyama et al., 2014; Coticchio et al., 2015; Sanfins et al., 2015). Published reports on IVM and IVF of primate (rhesus and marmoset) oocytes also vary in regard to gaseous environment, with either high oxygen for both IVM and IVF (Grupen et al., 1995; Gilchrist et al., 1997; Zheng et al., 2001; Schramm et al., 2003) or low for both these stages (Tomioka et al., 2012).

Although in vivo data on this issue is limited, especially concerning $\mathrm{O}_{2}$ tension in follicular fluid (FF), in general different stages of development from maturation of the oocyte within a follicle through its fertilization and early cleavage in the oviduct and finally to embryo implantation in uterus, occur under progressively reducing $\mathrm{O}_{2}$ tensions (Fischer and Bavister, 1993; Van Blerkom et al., 1997). To our knowledge, the only primate species for which follicular fluid (FF) $\mathrm{O}_{2}$ concentration has been published is human. Van Blerkom et al. (1997) showed that in human preovulatory follicles $(18-22 \mathrm{~mm}$ in diameter) $\mathrm{O}_{2}$ content varied from $<1$ to $5.5 \%$.

Van Blerkom (1998) also reported that $\mathrm{O}_{2}$ content in FF is highly variable both between different individuals and among follicles of the same individual and is associated with peri-follicular vascularity. A mathematic model published by Redding et al. (2008) suggests that mean levels of $\mathrm{O}_{2}$ in human $\mathrm{FF}$ during late antral and pre-ovulatory phases range between 11 and $51 \mathrm{mmHg}$ (1.5 to $6.7 \%$ ), and is therefore supportive of the results of Van Blerkom. Additionally, porcine $\mathrm{O}_{2}$ content in FF has been shown to have a similar level, 51 $\mathrm{mmHg}\left(6.7 \% ; \mathrm{O}_{2}\right.$ content was adjusted to corresponding percentage in gas by the authors with a correction for water vapor pressure $\left(47 \mathrm{mmHg}\right.$ by $\left.37^{\circ} \mathrm{C}\right)$ as follows: $\mathrm{O}_{2}$ tension $(\mathrm{mmHg})$ was divided by $760-47=713 \mathrm{mmHg}$ and multiplied by $100 \%$; Knudsen et al., 1978).

It must be noted that $\mathrm{O}_{2}$ concentration in biological liquids or in culture medium may be expressed either as actual concentration of dissolved $\mathrm{O}_{2}$ molecules in this liquid (vol\%), or, conventionally, as a partial pressure of $\mathrm{O}_{2}$ in a gas which would create such $\mathrm{O}_{2}$ concentration in a liquid (usually expressed in 
mmHg; Silbey et al., 2005). However, in the literature it is usual to present $\mathrm{O}_{2}$ content in the medium as vol\% of $\mathrm{O}_{2}$ in a gas phase above this medium rather than as vol $\% \mathrm{O}_{2}$ in the liquid itself; e.g. $\mathrm{O}_{2}$ concentration in pure water produced at $37^{\circ} \mathrm{C}$ and at $21 \mathrm{vol} \%(159 \mathrm{mmHg}) \mathrm{O}_{2}$ in air, is only up to $0.6 \mathrm{vol} \%$ (Weiss, 1970). Therefore, the values (\%) of $\mathrm{O}_{2}$ tension (or concentration) in the present manuscript actually mean vol\% of oxygen in the gas phase.

After ovulation, the matured oocyte surrounded by the expanded cumulus mass is drawn into the ampular end of the oviduct, where fertilization takes place. The only published $\mathrm{O}_{2}$ measurements for the reproductive tract of a primate species, is in rhesus monkey (Fischer and Bavister, 1993). They showed that during ovulation and in the early post-ovulatory phase, $\mathrm{O}_{2}$ concentration in the rhesus oviduct was around 56 $\mathrm{mmHg}(8 \%$; similar in ovulatory and non-ovulatory oviducts); but was much lower in the uterus, around 13 $\mathrm{mmHg}(1.8 \%)$. The same study provides information about $\mathrm{O}_{2}$ in rabbit and hamster reproductive tract (species with a bircornate uterus and different implantation/placentation strategies). It showed no reduction of $\mathrm{O}_{2}$ tension from oviduct to uterus in hamster (both $5.9 \%$ ) and only slight reduction in rabbit (7.4 and $5.9 \%$ in oviduct and uterus respectively). These species-specific differences strongly suggest that it would be advisable to measure $\mathrm{O}_{2}$ tension in both follicular fluid and the reproductive tract in the same species. Additional factors, such as uterus form (unicornuate $v s$. bicornuate) and implantation type (centric, eccentric or interstitial), may be associated with different oxygen tension patterns.

In most of the studies investigating $\mathrm{O}_{2}$ tension during IVM (with the only one study carried out in a primate species; Yeoman et al., 1999), effects are first observed at the early cleavage stage or even later - with alterations in blastocyst quality. Thus, $\mathrm{O}_{2}$ tension may be affecting some cytoplasmic factors and/or embryo genome activation; but this effect differed with species. For example, reduction of IVM $\mathrm{O}_{2}$ concentration from 20 to $5 \%$ improved subsequent blastocyst formation and/or quality in mice (Adam et al., 2004; Banwell et al., 2007; Preis et al., 2007) and porcine (Kikuchi et al., 2002; Iwamoto et al., 2005; Park et al., 2005), but negatively affected bovine oocyte and embryo development (Pinyopummintr and Bavister, 1995; Watson et al., 2000).

To our knowledge, to date, the only investigation of the effects of $\mathrm{O}_{2}$ on oocyte maturation in a primate species has been performed by Yeoman et al. (1999) for squirrel monkey. They evaluated the effect of an $\mathrm{O}_{2}$ - reduced environment and glucose on IVM outcome (in the presence of serum, gonadotrophins, pyruvate and glutamine). The present work, is the second involving a new world primate species and was performed as a part of a larger project to improve efficiency of normal mature oocyte production in vitro. With this aim, we evaluated the reasons for failure at each stage of culture to provide a background for improvement of in vitro conditions at next stages of experiments. Here we provide the first information on the effects of different $\mathrm{O}_{2}$ concentrations on IVM and IVF in the marmoset monkey, and is the first data of its type for any primate species.

\section{Materials and Methods}

All procedures were carried out according to German Animal Experimentation Law (Animal Experiment Permission \#33.42502/08-01.03) and animals were housed and surgery procedures carried out according to standard German Primate Centre practice for this species (Gilchrist et al., 1997; Isachenko et al., 2002).

Materials and Methods applied in the present paper have been previously published in full in Tkachenko et al. (2010) and therefore are described here as briefly as possible.

\section{Animals}

The females were housed with castrated partners and allowed to cycle naturally with ovarian cycles monitored by plasma progesterone levels to evaluate the mean length of luteal and follicular phase for each animal. Standard methods developed by Heistermann et al. (1993) were used. Animals were injected with prostaglandin F2 $\alpha$ analogue (PGF-2 $\alpha$, Estrumate, Essex Tierarznei, Munich, Germany) only on the cycle of the ovarectomy, in order to induce luteolysis and time the surgery. The females were operated 1-2 days before the expected day of ovulation.

Seven unstimulated, naturally cycling female marmosets were used for the present experiment. However, oocytes from one out of the seven animals demonstrated low maturation and failed to fertilize with a sperm of a proven male. This was assumed to be an animal-specific difference, and the data obtained from that animal has, therefore, been excluded from this study, with exception of the spindle defect data for the oocytes of that animal. The age of the six experimental animals varied from 26 to 30 months (mean age $29 \pm$ 2.5 months), weight - from 340 to $492 \mathrm{~g}(396 \pm 59 \mathrm{~g})$ and ovary volume - from 0.06 to $0.31 \mathrm{~cm}^{3}$ (mean $0.20 \pm$ $0.096 \mathrm{~cm}^{3}$; Ovary volume $[\mathrm{cm} 3]=$ length $[\mathrm{cm}] \times$ width $[\mathrm{cm}] \times$ height $[\mathrm{cm}])$. The animal excluded from the general statistics was 26 months old, $532 \mathrm{~g}$ (the youngest and heaviest individual), had mean ovary volume $0.18 \mathrm{~cm}^{3}$ and number of recovered COCs -39 . Between 17 and 68 COCs (mean $39 \pm 18$ COCs) suitable for culture were recovered from 1 full $+2 / 3$ ovaries of the six experimental animals, resulting in total 226 harvested COCs.

\section{Experimental design}

Three experimental groups were compared, each with a different combination of $\mathrm{O}_{2}$ concentrations during maturation and fertilization time: $8-5\left(8 \% \mathrm{O}_{2}\right.$ in maturation and $5 \%$ in fertilization); $20-5$ (20\% in maturation and 5\% in fertilization) and $20-20(20 \%$ in maturation and $20 \%$ in fertilization). As a part of a preliminary study we had tested maturation under $8 \%$ 
and fertilization under $20 \% \quad \mathrm{O}_{2} \quad$ (8-20 group). This additional group was included for first two animals, but excluded later from the main study in order not to sacrifice more animals due to failed fertilization under 8-20 conditions.

Material from each animal was processed individually with oocytes divided randomly and as equally as possible into the 3 treatment groups to equalize animal differences across the treatment groups. Oocytes from two out of six animals were divided into 4 treatment groups, including the 8-20 group, however, oocytes and embryos from different animals were never physically pooled.

\section{Media and culture plates}

All media, plate preparations and culture procedures have been performed as described in Tkachenko et al. (2015). IVM medium was supplemented with $1 \mu \mathrm{g} / \mathrm{ml} \quad \beta$-estradiol, as this concentration has been shown to be optimal according to the study cited above (Tkachenko et al., 2015).

\section{COCs recovery and IVM}

Recovered ovaries were carefully dissected into small pieces and antral follicles were punctured with forceps and 26G needles in ovary collection medium. Recovered COCs were immediately transferred into 4well plates with holding medium under oil and left in the incubator with controlled humidified atmosphere of $5 \% \mathrm{CO}_{2}$ in air, $38.3^{\circ} \mathrm{C}$ until the dissection of all ovary pieces was completed. Thereafter, COCs were randomly taken from all holding plates and equally divided between experimental groups with 5-7 COCs per well of IVM medium. After washing $\mathrm{COCs}$ were finally transferred into maturation wells $(25 \mu \mathrm{l})$ and matured in humidified atmosphere of either $5 \% \mathrm{CO}_{2}$ in air $(\sim 20 \%$ $\mathrm{O}_{2}$ ) or $5 \% \mathrm{CO}_{2}, 8 \% \mathrm{O}_{2}, 77 \% \mathrm{~N}_{2}$ at $38.3^{\circ} \mathrm{C}$ for $28-29 \mathrm{~h}$.

\section{Sperm collection and preparation}

Detailed procedures for sperm collection, preparation and IVF have been described previously in full (Valle et al., 2008; Tkachenko et al., 2015). For IVF sperm from donors with proven fertility was used. Each time, semen samples were collected from three sperm donors and the highest quality sample was chosen for IVF, based on sperm viability, motility and concentration.

\section{IVF}

One hour prior to IVF (28-29 $\mathrm{h}$ after IVM start) cumulus expansion of COCs was evaluated. Then after washing COCs were transferred into final drop of IVF medium $(25 \mu \mathrm{l})$ and $2 \mu \mathrm{l}$ of diluted sperm suspension with the final concentration of $20,000-30,000 \mathrm{sperm} / \mu \mathrm{l}$ was added to each drop. Oocytes were cultured with sperm for $15-16 \mathrm{~h}$ air at $38.3^{\circ} \mathrm{C}$ under one of the two experimental conditions - either in a gas mixture of
$5 \% \mathrm{CO}_{2}, 5 \% \mathrm{O}_{2}$ and $90 \% \mathrm{~N}_{2}$ or in $5 \% \mathrm{CO}_{2}$ in air $(\sim 20 \%$ $\mathrm{O}_{2}$ ). The day of IVF was called embryo day (ED) 0 .

\section{Embryo culture}

After IVF and fertilization status assessment performed as described in Tkachenko et al. ( 2015), fertilized oocytes/zygotes were cultured in a gas mixture of $5 \% \mathrm{CO}_{2}, 5 \% \mathrm{O}_{2}$ and $90 \% \mathrm{~N}_{2}$ with the half of the medium changed every $48 \mathrm{~h}$. Embryos were fixed in the case of no progress in the last $72 \mathrm{~h}$ or if visible signs of degeneration occurred, such as dark cytoplasm or loose cell contacts.

\section{Immunocytochemical procedures}

All oocytes arrested at different stages of maturation as well as arrested or degenerating embryos were fixed and stained for the evaluation of the final developmental stage and quality. Immunostaining procedures were carried out according to Delimitreva et al. (2006). Briefly, microtubules were stained using monoclonal anti- $\alpha$-tubulin mouse antibody (MP Biomedicals Germany GmbH, Eschwege, Germany 69$125,1: 50)$ and fluorescein isothiocyanate (1:100 FITCgoat anti-mouse immunoglobulin $G$ F(ab') 2Frag. Affin.). DNA was counterstained with Hoechst 33258 $(50 \mu \mathrm{g} / \mathrm{ml})$, added to the reaction mixture with FITC. Additionally, in embryos actin was stained with phalloidin-TRITC $(1 \mu \mathrm{g} / \mathrm{ml})$ added to the same reaction mixture. FITC: emission $525 \mathrm{~nm}$, excitation $490 \mathrm{~nm}$, Hoechst: emission $480 \mathrm{~nm}$, excitation $365 \mathrm{~nm}$; TRITC: emission $570 \mathrm{~nm}$, excitation $544 \mathrm{~nm}$.

\section{Evaluation of the results}

Cumulus expansion was evaluated after the end of maturation, before the COCs were transferred into fertilization medium, using 4 grades: 1) attached cumulus, 2) partially attached and expanded cumulus, 3) expanded cumulus with non-radial expansion, 4) cumulus with radial expansion (Tkachenko et al., 2010).

Developmental stage of arrested oocytes fixed after co-incubation with sperm was evaluated after fluorescent stain of microtubules and chromosomes. Oocyte stage was referred to as: germinal vesicle $(\mathrm{GV})$, metaphase I (MI), metaphase II (MII) or fertilized (only MII oocytes with evidence of fertilization, manifested by presence of 2 pronuclei and two distinct or fragmented polar bodies were counted as fertilized). Embryos were defined by the presence of at least two nucleated cells. Developmental rates were calculated both as progressive ( $\%$ from the previous stage) and from total number of non-degenerated oocytes. Embryo progression rate in the group was evaluated as a proportion of the embryos reached $2-3 ; 4-5 ; 6-8 ; 10-12$ cell and morula stage. Embryo morphology on ED2 for analysis of differences between the groups was evaluated by uniformity of cell size and visible nuclei distribution.

Final evaluation of embryo progression and quality was also performed after fluorescent staining. Normality of embryos was estimated by following criteria: all blastomeres have clear borders and 
cytoplasm, and single nucleus with normally dispersed interphase chromatin or mitotic chromosomal configuration, with less than $30 \%$ cytoplasmic fragmentation. Otherwise embryo was referred to as multinucleated or degenerated.

\section{Statistics}

Chi-square without Yates' correction or Fisherexact tests (SigmaStat 3.0) were used to assess the differences between experimental groups. P-value less than 0.05 was considered significant.

\section{Results}

\section{Cumulus expansion}

Cumulus expansion was compared for the oocytes matured under $8 \% \mathrm{O}_{2}$ and $20 \%$ (groups $20-20$ and 20-5 were pooled for this analysis). Only COCs with visible oocytes connected to surrounding cumulus cells were analyzed (64 and 134 COCs matured at 8 or $20 \%$, respectively, were evaluated). There was no difference in the rate of any expansion grade (Table 1).

Table 1. Effect of $\mathrm{O}_{2}$ concentration during IVM on cumulus expansion (data from 6 animals).

\begin{tabular}{lccccc}
\hline \multirow{2}{*}{$\begin{array}{l}\mathrm{O}_{2} \\
\text { concentration } \\
\text { at maturation }\end{array}$} & $\begin{array}{c}\text { attached (non- } \\
\text { expanded) }\end{array}$ & $\begin{array}{c}\text { partially attached and } \\
\text { expanded }\end{array}$ & $\begin{array}{c}\text { non-radial } \\
\text { expansion }\end{array}$ & $\begin{array}{c}\text { radial } \\
\text { expansion }\end{array}$ & Total COCs, \\
\hline $8 \%$ & $56.3 \pm 6.20(36)$ & $37.5 \pm 6.05(24)$ & $3.1 \pm 2.17(2)$ & $3.1 \pm 2.17(2)$ & 64 \\
\hline $20 \%$ & $44.0 \pm 4.29(59)$ & $39.6 \pm 4.22(53)$ & $10.4 \pm 2.64(14)$ & $6.0 \pm 2.05(8)$ & 134 \\
\hline
\end{tabular}

\section{Maturation and degeneration}

Since maturation status could be evaluated only after fertilization, the fact that some oocytes might have finished their maturation later, while being in fertilization medium, had to be taken into account. Therefore, maturation rate was evaluated separately in all 3 groups, without pooling together both groups matured under $20 \% \mathrm{O}_{2}$. MI progression rate in 20-20 group was significantly higher (91\%,
64/70) than in $8-5$ group $(78 \%, 50 / 64), \mathrm{P}<0.05$ (Fig. 1 ). The MI rate in 20-5 group was $89 \%$ (57/64). Contrastingly, the rates of progression towards MII stage, were significantly higher in $20-20$ group $(69 \%$, $48 / 70)$ than both in $20-5(45 \%, 29 / 64)$ and in $8-5(50 \%$, $32 / 64)$ groups $(\mathrm{P}<0.05)$.

Degeneration rates observed after $28 \mathrm{~h}$ of maturation did not differ significantly among the groups $(14 \%, 10 / 74 ; 16 \%, 12 / 76$ and $8 \%, 6 / 76$ in $8-5,20-5$ in 20-20 groups respectively.

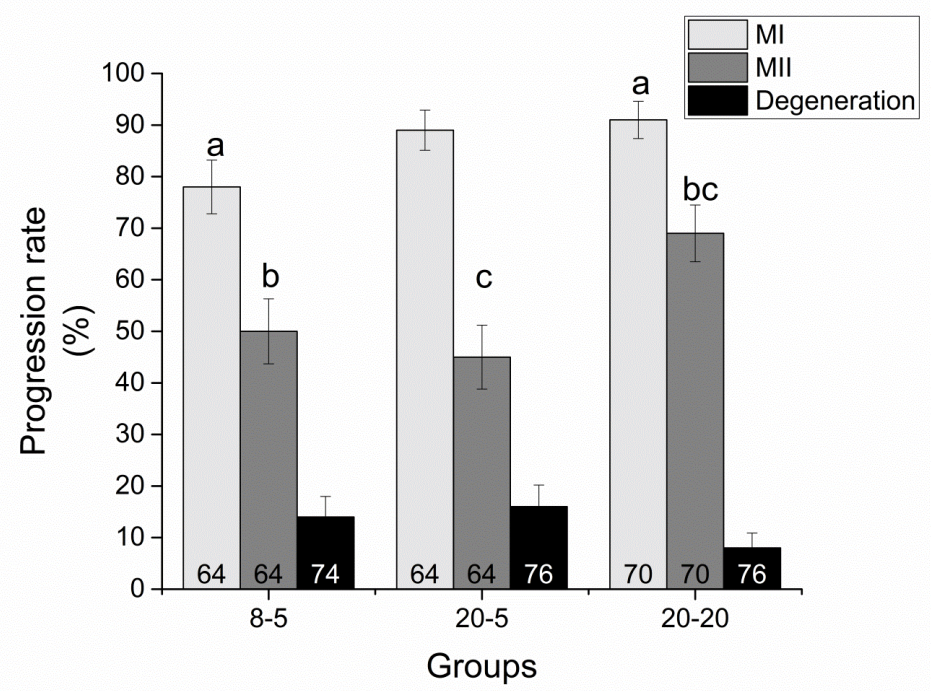

Figure 1. Maturation to MI (light grey bars), MII (dark grey bars) and degeneration (black bars). Numbers on the bars for MI and MII rates are total numbers of non-degenerated oocytes, and for degeneration rates - total number of all oocytes selected for IVM for each experimental group respectively. Group abbreviations $(8-5 ; 20-5 ; 20-20)$ : first figure is $\mathrm{O}_{2}$ tension at maturation, second - at fertilization. ${ }^{\mathrm{a}, \mathrm{b}, \mathrm{c}}$ Differences between columns with the same subscripts are significant $(\mathrm{P}<0.05)$.

\section{Fertilization}

In 20-5 group, fertilization occurred in $62 \%$ $(16 / 29)$ of the 29 oocytes which had reached MII, out of a total of 64 oocytes receiving this treatment. In the 20 20 group, out of 48 oocytes reaching MII from a total of 70 oocytes, $44 \%$ (21/48) fertilized. It shows that despite the high maturation rate the 20-20 group had lower proportional productivity in fertilization. In the 8-5 group, out of 64 oocytes total, only 32 matured and 17 fertilized $(53 \%)$. Importantly, rates of fertilization calculated from total initial oocyte number were similar for all three groups and ranged between 27 and 30\% in spite of differences in maturation rates. 
Contrastingly, oocytes matured under $8 \% \mathrm{O}_{2}$ and co-incubated with sperm under $20 \% \mathrm{O}_{2}$ failed to fertilize [data obtained with 13 oocytes from two animals: MI: 12/13 (92\%); MII: 8/13 (62\%)], although oocytes from these same two animals fertilized at the normal rate under the other conditions, indicating this was not an animal specific difference. Analysis of spindles in this group was performed (see below).

\section{Spindles}

Spindle shape was analyzed in 129 (both MI and MII) oocytes from 7 animals which failed to fertilize or failed to cleave after fertilization. These include also the data from the animal with total failure of fertilization, which did not demonstrate a difference in the pattern of spindle shape distribution compared with the other animals, and among all animals, there were no significant differences. Data on distribution of spindles into different classes is presented in Table 2.
Bipolar spindles with properly organized poles, well orientated microtubules and aligned chromosomes were referred to as apparently normal. However, this did not rule out possible minor abnormalities or chromosomal problems. Among the pooled MI+MII arrested oocytes, the highest rate of apparently normal spindles was observed in 20-20 groups (68 vs. $38 \%$ and $33 \%$ in $8-5$ and 20-5 groups, respectively, $\mathrm{P}<0.05)$. Although, no statistically significant difference was observed for comparisons between MI and MII oocytes from respective groups due to low numbers, this difference must be introduced by MI oocytes, for which normal spindle shape rate was $40 \%$ in $20-20$ group vs. 14 and 17 in two others, respectively. Additionally, analysis of spindles of the 8 oocytes from 8-20 group was performed, and high rates of abnormal spindles indicated development of cytoskeletal disturbances under these conditions (MI: $100 \%$ abnormal (2/2); MII: $67 \%$ abnormal (4/6)). Other $4 \mathrm{MI} / \mathrm{MII}$ oocytes from this group were lost during processing.

Table 2. Analysis of spindle shape in MI and MII arrested oocytes from different groups (data from 7 animals*).

\begin{tabular}{|c|c|c|c|c|c|c|c|c|c|c|c|}
\hline \multicolumn{4}{|c|}{ MI } & \multicolumn{4}{|c|}{ MII } & \multicolumn{4}{|c|}{ MI+MII pooled } \\
\hline \multirow{2}{*}{ Group } & \multicolumn{2}{|c|}{ Spindle shape, $\% \pm$ SD (n) } & \multirow{2}{*}{ Total } & \multirow{2}{*}{ Group } & \multicolumn{2}{|c|}{ Spindle shape, $\% \pm \mathrm{SD}(\mathrm{n})$} & \multirow{2}{*}{ Total } & \multirow{2}{*}{ Group } & \multicolumn{2}{|c|}{ Spindle shape, $\% \pm \mathrm{SD}(\mathrm{n})$} & \multirow{2}{*}{ Total } \\
\hline & Normal & Abnormal & & & Normal & Abnormal & & & Normal & Abnormal & \\
\hline $8-5$ & $14 \pm 7.6(3)$ & $86 \pm 7.6(18)$ & 21 & $8-5$ & $62 \pm 10.6(13)$ & $38 \pm 10.6(8)$ & 21 & $8-5$ & $38 \pm 25.6(16)^{a}$ & $62 \pm 25.6(26)$ & 42 \\
\hline $20-5$ & $17 \pm 6.9(5)$ & $83 \pm 6.9(25)$ & 30 & $20-5$ & $75 \pm 12.5(9)$ & $25 \pm 12.5(3)$ & 12 & $20-5$ & $33 \pm 19.9(14)^{\mathrm{a}}$ & $68 \pm 19.9(28)$ & 42 \\
\hline $20-20$ & $40 \pm 12.6(6)$ & $60 \pm 12.6(9)$ & 15 & $20-20$ & $73 \pm 8.1(22)$ & $27 \pm 8.1(8)$ & 30 & $20-20$ & $62 \pm 11.4(28)^{b}$ & $38 \pm 11.4(17)$ & 45 \\
\hline
\end{tabular}

*Oocytes from the animal excluded from the main analysis due to failed fertilization were included into this analysis. 8-5; 20-5; 20-20: in group abbreviations first figure is $\mathrm{O} 2$ tension at maturation, second - at fertilization. ${ }^{\mathrm{a}, \mathrm{b}}$ Different subscripts indicate significant $(\mathrm{P}<0.05)$ differences.

\section{First cleavage}

Significantly lower proportion of fertilized oocytes achieved first cleavage in $8-5$ group $(59 \%$, $10 / 17, \mathrm{P}<0.05)$ compared with $20-20$ group $(95 \%$,
$20 / 21)$, and $83 \%(15 / 18)$ of fertilized oocytes cleaved in 20-5 group. No difference was observed in total embryo production assessed based on the proportions calculated from total initial numbers of oocytes per group (Fig.2).

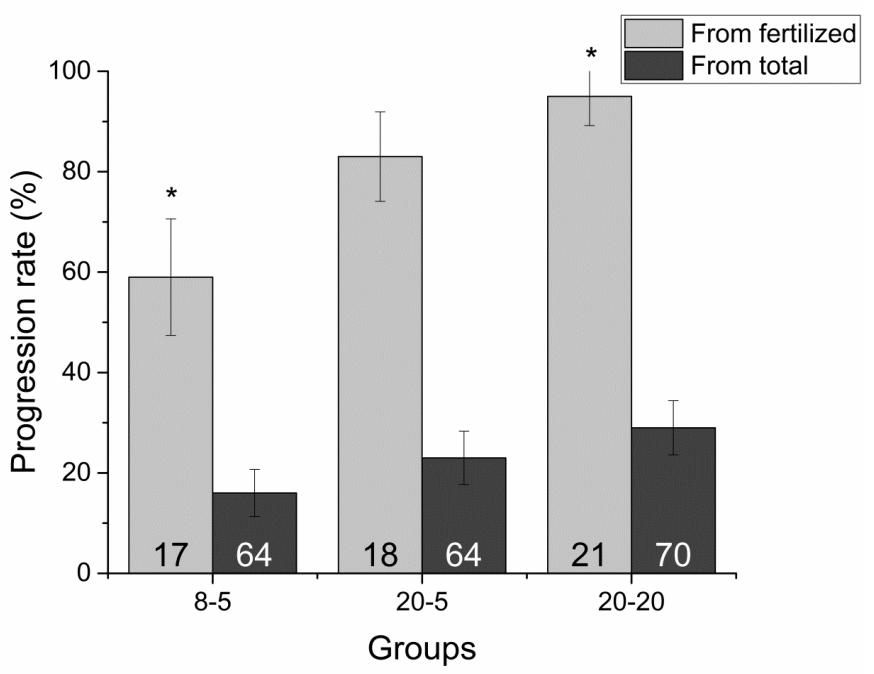

Figure 2. Cleavage rate from fertilized oocytes (light grey bars) and from total non-degenerated (dark grey bars). Numbers on the bars are the totals from which each rate has been calculated (fertilized or total non-degenerated, respectively). Group abbreviations $(8-5 ; 20-5 ; 20-20)$ : first figure is $\mathrm{O}_{2}$ tension at maturation, second - at fertilization. ${ }^{*}$ The asterisk marks significant differences between the columns $(\mathrm{P}<0.05)$. 


\section{Embryo progression and embryo quality}

There was a consistent pattern suggesting better quality of embryos in the groups matured under high oxygen. Analysis of embryo morphology on ED2 (when embryos normally have 4 cells) demonstrated a trend for better embryo morphology in groups matured under high oxygen (both 60\%: $9 / 15$ and 12/20 in 20-5 and 20-20 groups, respectively). Examples of good $v s$. bad morphology embryos are shown in Tkachenko et al. (2010). We have, however, noted a trend towards better progression of 4-cell embryos to morula stage in $20-5$ group $(30 \%, 3 / 10$ vs. $12.5 \%$ in both other groups: $2 / 16$ in $20-20$ and $1 / 8$ and in $8-5$ groups) compared to $8-5$ group (only $20 \%$ of embryos had normal morphology, $2 / 10$ ). Example of an individual embryo progression to compact morula is shown in Fig.3. One morula from 205 group and one from 20-20 group have demonstrated evidence of early antrum formation, however, they arrested at this stage (see these early blastocysts in Fig.4).

Results of evaluation of micronucleation in fixed embryos of all stages have shown a similar pattern. The highest rate of apparently normal embryos $(50 \%, 7 / 14)$ was in $20-5$ group the lowest - in 8-5 group $(22 \%, 2 / 9)$ (Table 3$)$.
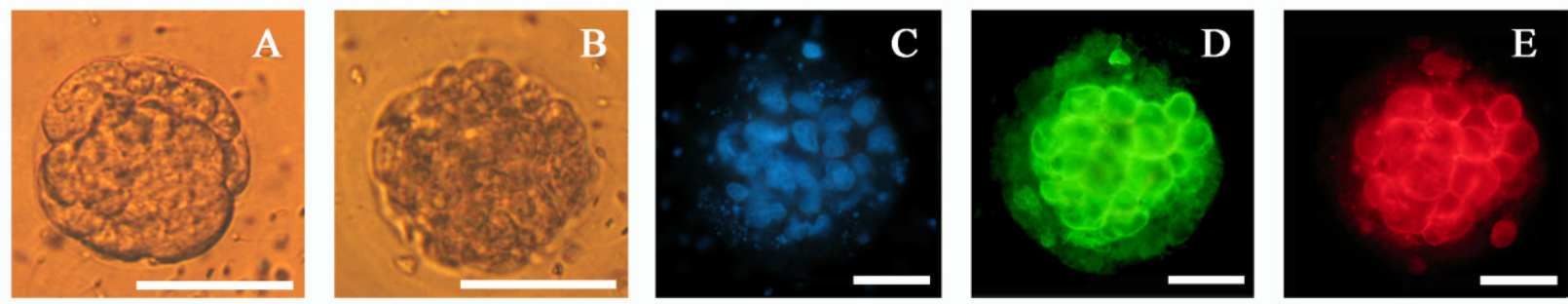

Figure 3. An example of an embryo developed to morula stage on embryo day (ED) 8 (A) and ED 9 (B). This embryo was from the group matured at $20 \% \mathrm{O} 2$ and fertilized at $5 \% \mathrm{O}_{2}$ Nuclei of approx. 25 cells are visible. Stained (after fixation on ED 11) with Hoechst (B), tubulin (C) and actin (D). Strong localization of actin and tubulin at cell junctions indicates that compaction has occurred. Scale bars are $70 \mu \mathrm{m}(\mathrm{A}, \mathrm{B})$ and $50 \mu \mathrm{m}(\mathrm{C}, \mathrm{D}, \mathrm{E})$.
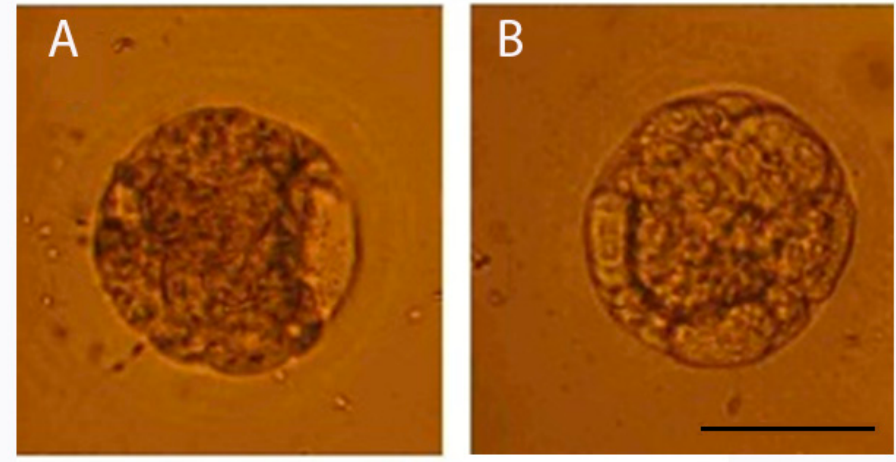

Figure 4. Early blastocysts developed in culture (embryo day 9) after IVM under $20 \%$ and IVF under 5\% O2 (A), and after IVM under 20\% and IVF under $20 \%$ O2 (B). Scale bar is $70 \mathrm{um}$.

Table 3. Multinucleation in embryos. Embryos from 4 animals were stained for tubulin and chromatin visualization.

\begin{tabular}{lccc}
\hline \multirow{2}{*}{ Embryo quality } & \multicolumn{3}{c}{ Rates of embryos with different quality, \% \pm SD (n) } \\
\cline { 2 - 4 } & $8-5$ & $20-5$ & $20-20$ \\
\hline Apparently normal & $22 \pm 13.8(2)$ & $50 \pm 13.4(7)$ & $41 \pm 11.9(7)$ \\
Multinucleated & $33 \pm 15.7(3)$ & $43 \pm 13.2(6)$ & $41 \pm 11.9(7)$ \\
Degenerated & $45 \pm 16.5(4)$ & $7 \pm 6.8(1)$ & $18 \pm 9.0(3)$ \\
Embryos total, $\mathrm{n}$ & 9 & 14 & 17 \\
\hline
\end{tabular}

$8-5 ; 20-5 ; 20-20$ : in group abbreviations first figure is $\mathrm{O}_{2}$ tension at maturation, second - at fertilization.

\section{Discussion}

The impact of $\mathrm{O}_{2}$ concentration during IVM and IVF on oocyte maturation, fertilization, and subsequent embryo development has been investigated for marmoset monkey oocytes, and this provides the first information of this kind for this species.

The present study demonstrates better results after maturation under 20 than $8 \%$ gas phase $\mathrm{O}_{2}$. This is similar to the results obtained for other species (Pinyopummintr and Bavister, 1995; Watson et al., 2000; Kikuchi et al., 2002; Oyamada and Fukui, 2004;
Iwamoto et al., 2005; Park et al., 2005; Banwell et al., 2007; Mingoti et al., 2009), including the only other comparable study for primates (squirrel monkey) published by Yeoman et al. (1999).

These findings seem surprising, since culture under atmospheric $\mathrm{O}_{2}$ would actually result in $\mathrm{O}_{2}$ tension in culture drops significantly higher than the natural oocyte environment during periovulatory maturation within the follicle: reported values in human and porcine $\mathrm{FF}$ correspond to about $6 \% \mathrm{O}_{2}$ in the gas phase (Knudsen et al., 1978; Fischer and Bavister, 1993; Van Blerkom et al., 1997; Redding et al., 2008). 
However, there are several factors of the culture environment which may either actually lower the amount of $\mathrm{O}_{2}$ available for the oocytes or protect them from excessive oxidation. First, $\mathrm{O}_{2}$ consumption by cumulus cells may act as a natural buffer protecting the oocyte from too high $\mathrm{O}_{2}$. Cumulus complex has been estimated to reduce $\mathrm{O}_{2}$ level at the oocyte surface to 5$15 \%$ lower than in surrounding FF (Clark et al., 2006). Additionally, when culture is performed in microdrops under mineral oil, the amount of $\mathrm{O}_{2}$ available in a culture drop might be further slightly reduced due to slower $\mathrm{O}_{2}$ diffusion into medium and geometry of the drop (Stokes, 2009). There are also other factors, such as culture medium components, which may mitigate effects of high $\mathrm{O}_{2}$ on oocyte metabolism. All this transforms what first appears to be a nonphysiologically high $\mathrm{O}_{2}(20 \%)$ into one closer to physiological at the surface of the oocyte in our culture conditions. It is therefore logical that a lower $\mathrm{O}_{2}$ in the gas phase (e.g. $8 \%$ ), could produce hypoxic conditions in the culture drop through the same mechanisms, and damage the oocyte similar to the changes occurring in oocytes in atretic follicles with inadequate vascularity. This idea is further supported by in vivo data demonstrating that healthy preovulatory follicles have higher $\mathrm{O}_{2}$ content in FF compared to smaller or less well vascularised follicles (Van Blerkom et al., 1997). This study further showed that oocytes from follicles with $\mathrm{O}_{2}$ content less than 3\% appeared to possess high rates of abnormal chromosome organization and alignment (Van Blerkom et al., 1997). Our analysis of spindle organization in arrested oocytes indirectly supports this finding.

Further, we observed the highest rate of normal spindles in the group matured and fertilized under 20\% oxygen, which exceeded the rate in 20-5 group. In our study maturation status of oocytes could be evaluated only post-IVF, once cumulus cells were removed. Therefore by the time of assessment, mature oocytes could have included those which completed polar body extrusion in fertilization medium. We found here a significant difference in MII progression rates $(69 v s$. $45 \%$ ) between two groups cultured under $20 \% \quad \mathrm{O}_{2}$ during IVM and either 20 or $5 \% \mathrm{O}_{2}$ during IVF. The effect of gas during fertilization must be attributed to the completion of maturation among those oocytes which had not fully matured in IVM medium, which also explains the higher rate of MII progression in the 20-20 group compared to the 20-5 group. Apparently, drastic reduction in oxygen tension (for example from 20 down to 5\%) impairs late maturation causing disturbances in cytoskeletal organization. In support of this, negative effect was also observed for the group matured under low and fertilized under high oxygen, evidenced by high rates of spindle abnormalities and absence of fertilization.

In this study we have achieved the aim of improving early stages on in vitro culture of unstimulated marmoset oocytes, obtaining high rates of maturation, fertilization and early cleavage. However, the next stages of the experiments need to focus on improvements of the progressive embryo development using our best maturation and fertilization conditions and varying the embryo culture media in a focused experimental design. It should be noted that production of blastocysts from immature oocytes collected from unstimulated marmosets has been only reported by two groups so far. Tomioka et al. obtained 13 morulas from starting number of 428 oocytes (12 animals), while Gilchrist reported 31 morulas from 135 oocytes (3 animals; (Gilchrist et al., 1997; Tomioka et al., 2012). In this study we started with 226 oocytes from 6 animals, and obtained in total 6 morulas. However, unlike Tomioka et al., and similar to Gilchrist et al. (who used the same embryo culture system as in this paper), progression of morulas to early blastocysts in the present study was low. Porcine culture medium used by Tomioka et al. for embryo culture definitely supports morula to blastocyst transition better than our culture medium, and analysis of these two culture approaches might provide valuable information on marmoset embryo requirements.

In summary, in terms of oocyte maturation, early embryo development and embryo quality, $20 \% \mathrm{O}_{2}$ during IVM of marmoset monkey oocytes provided better results than $8 \% \mathrm{O}_{2}$ under the present culture conditions, while lower $\mathrm{O}_{2}$ seemed to be preferable during IVF based on embryo quality compared with $20 \%$ even though $20 \% \mathrm{O}_{2}$ appears to have allowed late maturing oocytes to complete the process in the fertilization medium. These results provide the first information of its kind for this species, and are an important step in the process of developing an optimized IVM-IVF-embryo development system for marmoset monkey, but further studies are required.

\section{Acknowledgments}

The authors wish to thank K. Fuhrmann, A Berenson and N. Umland for blood sampling, cycle monitoring, sperm collection and technical help during operations and laboratory work. We are thankful to T. Becker and J. Lademann for surgery and to Dr. M. Heistermann, A. Heistermann and J. Hagedorn for hormone assay services. The authors are thankful for some helpful comments in physics from Dr. K.A. Chishko (B.Verkin Institute for Low Temperature Physics and Engineering of the National Academy of Sciences of Ukraine, Kharkiv, Ukraine).

O.Y. Tkachenko was supported in part by a DAAD and a Young Scientists grant from the German Primate Center. Travel expenses to work in DPZ for S. Delimitreva were paid by the Bulgarian Ministry in Education and Science, Grant \# VU-L-311/07.

This study was carried out by O.Y. Tkachenko in partial fulfilment of the degree of PhD.

\section{References}

Adam AA, Takahashi Y, Katagiri S, Nagano M. 2004. Effects of oxygen tension in the gas atmosphere during in vitro maturation, in vitro fertilization and in vitro culture on the efficiency of in vitro production of mouse embryos. Jap J Vet Res, 52:77-84. 
Banwell KM, Lane M, Russell DL, Kind KL, Thompson JG. 2007. Oxygen concentration during mouse oocyte in vitro maturation affects embryo and fetal development. Hum Reprod, 22:2768-2775.

Bavister BD. 1995. Culture of preimplantation embryos: facts and artifacts. Hum Reprod Update, 1:91148.

Clark AR, Stokes YM, Lane M, Thompson JG. 2006. Mathematical modelling of oxygen concentration in bovine and murine cumulus-oocyte complexes. Reproduction, 131:999-1006.

Coticchio G, Dal Canto M, Guglielmo MC, Albertini DF, Renzini MM, Merola M, Lain M, Sottocornola M, De Ponti E, Fadini R. 2015. Double-strand DNA breaks and repair response in human immature oocytes and their relevance to meiotic resumption. J Assist Reprod Genet, 32:1509-1516.

Delimitreva S, Zhivkova R, Isachenko E, Umland N, Nayudu PL. 2006. Meiotic abnormalities in in vitromatured marmoset monkey (Callithrix jacchus) oocytes: development of a non-human primate model to investigate causal factors. Hum Reprod, 21:240-247.

Fischer B, Bavister BD. 1993. Oxygen tension in the oviduct and uterus of rhesus monkeys, hamsters and rabbits. J Reprod Fertil, 99:673-679.

Gelatt KN, van der Woerdt A, Ketring KL, Andrew SE, Brooks DE, Biros DJ, Denis HM, Cutler TJ. 2001. Enrofloxacin-associated retinal degeneration in cats. Vet Ophthalmol, 4:99-106.

Gilchrist RB, Nayudu PL, Hodges JK. 1997. Maturation, fertilization, and development of marmoset monkey oocytes in vitro. Biol Reprod, 56:238-246.

Grupen CG, Nagashima H, Nottle MB. 1995. Cysteamine enhances in vitro development of porcine oocytes matured and fertilized in vitro. Biol Reprod, 53:173-178.

Heistermann M, Tari S, Hodges JK. 1993. Measurement of faecal steroids for monitoring ovarian function in New World primates, Callitrichidae. $J$ Reprod Fertil, 99:243-251.

Isachenko EF, Nayudu PL, Isachenko VV, Nawroth F, Michelmann HW. 2002. Congenitally caused fused labia in the common marmoset (Callithrix jacchus). $J$ Med Primatol, 31:350-355.

Iwamoto M, Onishi A, Fuchimoto D, Somfai T, Takeda K, Tagami T, Hanada H, Noguchi J, Kaneko H, Nagai T, Kikuchi K. 2005. Low oxygen tension during in vitro maturation of porcine follicular oocytes improves parthenogenetic activation and subsequent development to the blastocyst stage. Theriogenology, 63:1277-1289.

Karja NW, Wongsrikeao P, Murakami M, Agung B, Fahrudin M, Nagai T, Otoi T. 2004. Effects of oxygen tension on the development and quality of porcine in vitro fertilized embryos. Theriogenology, 62:1585-1595. Kikuchi K, Onishi A, Kashiwazaki N, Iwamoto M, Noguchi J, Kaneko H, Akita T, Nagai T. 2002. Successful piglet production after transfer of blastocysts produced by a modified in vitro system. Biol Reprod, 66:1033-1041.

Knudsen JF, Litkowski LJ, Wilson TL, Guthrie HD, Batta SK. 1978. Concentrations of hydrogen ions, oxygen, carbon dioxide and bicarbonate in porcine follicular fluid. $J$ Endocrinol, 79:249-250.

Kovacic B, Vlaisavljevic V. 2008. Influence of atmospheric versus reduced oxygen concentration on development of human blastocysts in vitro: a prospective study on sibling oocytes. Reprod Biomed Online, 17:229-236.

Koyama K, Kang SS, Huang W, Yanagawa Y, Takahashi Y, Nagano M. 2014. Aging-related changes in in vitro-matured bovine oocytes: oxidative stress, mitochondrial activity and ATP content after nuclear maturation. J Reprod Dev, 60:136-142.

Meintjes M, Chantilis SJ, Douglas JD, Rodriguez AJ, Guerami AR, Bookout DM, Barnett BD, Madden JD. 2009. A controlled randomized trial evaluating the effect of lowered incubator oxygen tension on live births in a predominantly blastocyst transfer program. Hum Reprod, 24:300-307.

Mingoti GZ, Caiado Castro VS, Meo SC, Barretto LS, Garcia JM. 2009. The effect of interaction between macromolecule supplement and oxygen tension on bovine oocytes and embryos cultured in vitro. Zygote, 17:321-328.

Oyamada T, Fukui Y. 2004. Oxygen tension and medium supplements for in vitro maturation of bovine oocytes cultured individually in a chemically defined medium. J Reprod Dev, 50:107-117.

Park JI, Hong JY, Yong HY, Hwang WS, Lim JM, Lee ES. 2005. High oxygen tension during in vitro oocyte maturation improves in vitro development of porcine oocytes after fertilization. Anim Reprod Sci, 87:133-141.

Pinyopummintr T, Bavister BD. 1995. Optimum gas atmosphere for in vitro maturation and in vitro fertilization of bovine oocytes. Theriogenology, 44:471477.

Piotrowska-Nitsche K, Yang SH, Banta H, Chan AW. 2009. Assisted fertilization and embryonic axis formation in higher primates. Reprod Biomed Online, 18:382-390.

Preis KA, Seidel GE, Jr., Gardner DK. 2007. Reduced oxygen concentration improves the developmental competence of mouse oocytes following in vitro maturation. Mol Reprod Dev, 74:893-903.

Redding GP, Bronlund JE, Hart AL. 2008. Theoretical investigation into the dissolved oxygen levels in follicular fluid of the developing human follicle using mathematical modelling. Reprod Fertil Dev, 20:408-417.

Sanfins A, Plancha CE, Albertini DF. 2015. Preimplantation developmental potential from in vivo and in vitro matured mouse oocytes: a cytoskeletal perspective on oocyte quality. J Assist Reprod Genet, 32:127-136.

Schramm RD, Paprocki AM, VandeVoort CA. 2003. Causes of developmental failure of in-vitro matured rhesus monkey oocytes: impairments in embryonic genome activation. Hum Reprod, 18:826-833.

Silbey RJ, Alberty RA, Bawendi MG. 2005. Physical Chemistry. New York, NY: John Wiley.

Stokes YM. 2009. Quantifying oxygen diffusion in paraffin oil used in oocyte and embryo culture. Mol 
Reprod Dev, 76:1178-1187.

Tkachenko OY, Delimitreva S, Isachenko E, Valle RR, Michelmann HW, Berenson A, Nayudu PL. 2010. Epidermal growth factor effects on marmoset monkey (Callithrix jacchus) oocyte in vitro maturation, IVF and embryo development are altered by gonadotrophin concentration during oocyte maturation. Hum Reprod, 25:2047-2058.

Tkachenko OY, Delimitreva S, Heistermann M, Scheerer-Bernhard JU, Wedi E, Nayudu PL. 2015. Critical estradiol dose optimization for oocyte in vitro maturation in the common marmoset. Theriogenology, 83:1254-1263.

Tomioka I, Takahashi T, Shimada A, Yoshioka K, Sasaki E. 2012. Birth of common marmoset (Callithrix jacchus) offspring derived from in vitro-matured oocytes in chemically defined medium. Theriogenology, 78:1487-1493.

Valle RR, Valle CM, Nichi M, Muniz JA, Nayudu PL, Guimaraes MA. 2008. Validation of nonfluorescent methods to reliably detect acrosomal and plasma membrane integrity of common marmoset (Callithrix jacchus) sperm. Theriogenology, 70:115120.

Van Blerkom J, Antczak M, Schrader R. 1997. The developmental potential of the human oocyte is related to the dissolved oxygen content of follicular fluid: association with vascular endothelial growth factor levels and perifollicular blood flow characteristics. Hum Reprod, 12:1047-1055.

Van Blerkom J. 1998. Epigenetic influences on oocyte developmental competence: perifollicular vascularity and intrafollicular oxygen. $J$ Assist Reprod Genet, 15:226-234.

Watson AJ, De Sousa P, Caveney A, Barcroft LC, Natale D, Urquhart J, Westhusin ME. 2000. Impact of bovine oocyte maturation media on oocyte transcript levels, blastocyst development, cell number, and apoptosis. Biol Reprod, 62:355-364.

Weiss RF. 1970. The solubility of nitrogen, oxygen, and argon in water and seawater. Deep-Sea Res, 17:721735 .

Yeoman RR, Williams LE, Abee CR. 1999. Low oxygen inhibits but complex high-glucose medium facilitates in vitro maturation of squirrel monkey oocyte-granulosa cell complexes. J Assist Reprod Genet, 16:102-107.

Zheng P, Wang H, Bavister BD, Ji W. 2001. Maturation of rhesus monkey oocytes in chemically defined culture media and their functional assessment by IVF and embryo development. Hum Reprod, 16:300305. 